\title{
The Influence of Inclined Magnetic Field and Heat Transfer on the MHD Convective Flow in a Vertical Channel Filled Partly with Porous Medium
}

\section{P. T. Hemamalini, M. Shanthi}

\begin{abstract}
An analyticstudy has been madeof a laminar fully developed MHD flow bounded by infinite vertical parallel plates with effect of inclined magnetic fieldpartly filled with fluid and partly with porous matrix. The motions of the plates are in the opposite direction and are maintained at distinct temperatures. The perturbation method has been chosen to derive the expression for velocity flow and temperaturedistributionand the effect on flow velocity and temperature due to magnetic field and Darcy number has been illustrated for the fluid and porous region with the helpof graph.
\end{abstract}

KEYWORDS:MHD Convection Flow,Inclined Magnetic Field, Vertical Channel, Darcy number, partly filled porous medium.

\section{INTRODUCTION}

Asignificant interest has been created by recent technological implications in the study of flow in channels through partly filled porous medium. An extensive research has been carried out in the multiphase flow through channels and different wall temperatures in thepresence of inclined magnetic field. Due to its common occurrence in engineering fields such as in thermal energy storage system,petrol purification,etc., itwould be interesting to study the mhd flow in a region with partly loaded with porous medium in thepresence of inclined magnetic field.

A. K. Singh, and T.Paul (2006) considered transient natural convection between two vertical walls heated/cooled asymmetrically. M.M.S. El-Din (2007) investigated on the fully developed mixed convection laminar flow with viscous dissipation in a uniformly heated vertical double-passage channel. Radiation effects on an unsteady MHD convective heat and mass transfer flow past a semi-infinite vertical permeable moving plate embedded in a porous medium was illustrated by V.R. Prasad and N.B. Reddy,(2008).D.S.Chauhan and V. Kumar,(2009)investigated on the effects of slip conditions on forced convection and entropy generation in a circular channel occupied by a highly porous medium: Darcy extended Brinkman-Forchheimer model. Srivastava and Singh.A. K., (2010) considered the mixed convection in a composite system bounded by vertical walls.

Revised Manuscript Received on October 22, 2019

* Correspondence Author

Dr.P.T.Hemamalini, Department of Mathematics, Karpagam Academy of Higher Education, TamilNadu,India,hema_0869@yahoo.co.in

M.Shanthi, Research Scholar,Department of Mathematics,Karpagam Academy of Higher Education,TamilNadu,India,shanthi2saba@gmail.com
Free convection flow through vertical plates moving in different direction and partially filled with porous medium was discussed by Umesh Gupta, et al, (2011). Magnetohydrodynamic convection effects with viscous and ohmic dissipation in a vertical channel partially filled by a porous medium was illustrated by Dileep Singh Chauhan and Rashmi Agrawal,(2012). Prasad, K. V., K. Vajravelu, et al, (2013) studied MHD flow with heat transfer in a powerlaw liquid film at a porous surface in the presence of thermal radiation. S. Purkayastha and Choudhary .R(2014)considered visco-elastic effects on convection flow through a vertical rotating channel partially filled with a porous medium. Adeyemi Isaiah Fagbade, et al(2015) observed the influence of magnetic field, viscous dissipation and thermophoresis on Darcy-Forcheimer mixed convection flow in fluid saturated porous media. Free convection flow of a jeffrey fluid between vertical plates partially filled with porous medium was learnt by $\mathrm{S}$. Dhananjaya1, et al(2015),A. Adeniyan ,et al,(2016)discussedmixed convection radiating flow and heat transfer in a vertical channel partially filled With a DarcyForchheimer Porous Substrate. V. G. Gupta, Ajay Jain, et al.,(2016) studied the convective effects on MHD flow and heat transfer between vertical plates moving in opposite direction and partially filled with a porous medium. Heat, Mass Transfer in free Convective Flow of Walter's Liquid Model-B through Rotating Vertical Channel was illustrated byPooja Sharma, et al.(2017).

The aim of the study is to analyze the fully developed laminar flow in the vertical channel partially porous medium and clear fluid in presences of inclined magnetic field.

\section{PHYSICAL DESCRIPTION OF THE PROBLEM}

A vertical channel of width $\mathrm{H}$, partly loaded with clear fluid and partially with porous matrix having interface, is considered with inclined magnetic field, when one of the plates is heated and the other is cooled.The fully developedlaminarfree- convection flow confined in the vertical channel is illustrated.The Cartesian co-ordinate system is sotaken that $\mathrm{X}$ axis ismarked along one of the plates but opposite to the gravitational field and $\mathrm{Y}$ axis perpendicular to it. The verticalplates are placed at $\lfloor\bar{Y}=0\rfloor$, and $[\bar{Y}=H]$ are moving in opposite directions with the velocities $\bar{U}_{f}$ and $\bar{U}_{p}$ in the $\mathrm{X}$ direction.Let the wall temperature at $\bar{Y}=0$ be $\bar{T}_{f}=\left[\bar{T}_{c}+A\left(\bar{T}_{h}-\bar{T}_{c}\right)\right] \quad$ and 
$\bar{Y}=H$ be $\bar{T}_{p}=\left[\bar{T}_{c}+B\left(\bar{T}_{h}-\bar{T}_{c}\right)\right]$ respectively.
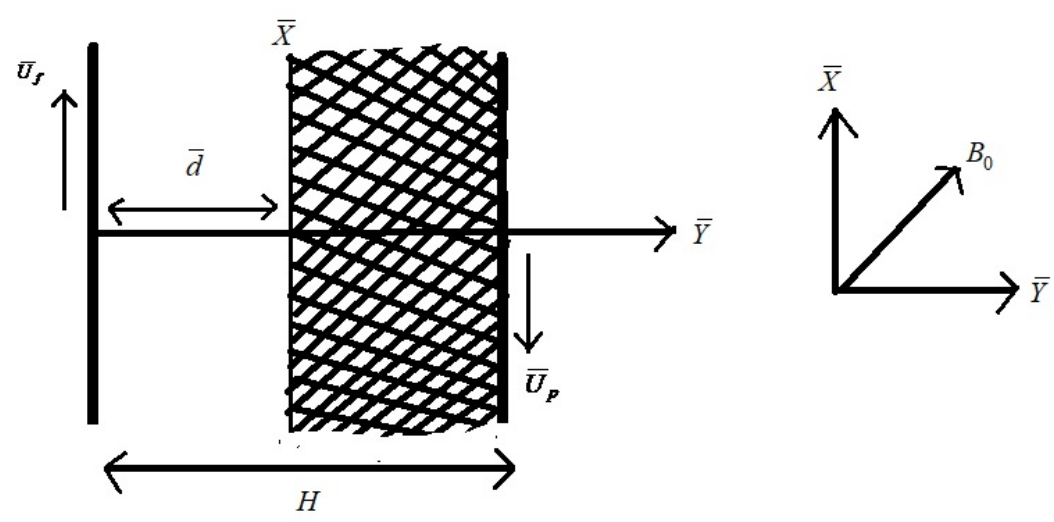

Figure 1 Geometric model and coordinate system

\section{BASIC EQUATIONS AND METHODOLOGY}

The governing equations for the above physical configuration are illustrated by:

Clear Fluid Region

$$
\frac{\partial^{2} \bar{U}_{f}}{\partial \bar{y}^{2}}+\frac{g \beta}{v}\left(\bar{T}_{f}-\bar{T}_{c}\right)-\frac{B_{0} \sigma}{\rho v} \sin \theta \bar{U}_{f}=0
$$

(1)

$$
\left(\frac{d \bar{U}_{f}}{d \bar{y}}\right)^{2}+\frac{k}{v} \frac{d^{2}}{d \bar{y}^{2}}\left(\bar{T}_{f}-\bar{T}_{c}\right)=0
$$

Porous fluid Region

$$
\begin{aligned}
& \frac{\partial^{2} \bar{U}_{p}}{\partial \bar{y}^{2}}+\frac{g \beta}{v}\left(\bar{T}_{p}-\bar{T}_{c}\right)-\frac{B_{0} \sigma}{\rho v} \sin \theta \bar{U}_{p}-\frac{\bar{U}_{p}}{\bar{k}}=0 \\
& \left(\frac{d \bar{U}_{p}}{d \bar{y}}\right)^{2}+\frac{k}{v} \frac{d^{2}}{d \bar{y}^{2}}\left(\bar{T}_{p}-\bar{T}_{c}\right)-\frac{\bar{U}_{p}^{2}}{\bar{k}}=0
\end{aligned}
$$

The initial,boundary conditions are:

$$
\begin{array}{lll}
\bar{U}_{f}=\frac{g \beta H^{2}\left[\bar{T}_{h}-\bar{T}_{c}\right]}{v} u_{0} ; \bar{T}_{f}=\left[\bar{T}_{c}+A\left(\bar{T}_{h}-\bar{T}_{c}\right)\right] & \text { at } & \bar{y}=0 \\
\bar{U}_{p}=\frac{-g \beta\left[\bar{T}_{h}-\bar{T}_{c}\right] H^{2}}{v} u_{0} ; \bar{T}_{p}=\left[\bar{T}_{c}+B\left(\bar{T}_{h}-\bar{T}_{c}\right)\right] & \text { at } & \bar{y}=H \\
\frac{d \bar{U}_{f}}{d \bar{y}}=\frac{d \bar{U}_{p}}{d \bar{y}} ; \bar{U}_{f}=\bar{U}_{p} ; \frac{d \bar{T}_{f}}{d \bar{y}}=\frac{d \bar{T}_{p}}{d \bar{y}} \overline{T_{f}}=\bar{T}_{p} ; & \text { at } & \bar{y}=\bar{d}
\end{array}
$$

Where $\bar{U}_{f}$-denotes the velocity of the plate at $(\mathrm{Y}=0), \bar{U}_{p}$ plate velocity at $(\mathrm{Y}=\mathrm{H}), \mathrm{B}_{0}$ strength of uniform magnetic field, $\rho$ density and $k$ thermal conductivity. The thermalexpansion co-efficient of the fluid is marked by $\beta$, thermal Grashof number by $\mathrm{G}_{\mathrm{r}}$, temperature of the heated plate by $\bar{T}_{h}$, temperature of the cooled plate by $\bar{T}_{c}$ and the acceleration due to gravity by $g$, A and B are the interchangeable temperatures at the plates. $\sigma$ denotes the electrical conductivity,the permeability of porous medium is marked by $\bar{k}$ andKis thethermal conductivity and Nis the buoyancy parameter. Kinematic viscosity and the dynamic viscosity are marked by $\mu$ and vrespectively.

Introducing non-dimensional quantities,

$$
\begin{aligned}
& U_{f}=\frac{v \bar{U}_{f}}{g \beta H^{2}\left(\bar{T}_{h}-\bar{T}_{c}\right)}, \theta_{f}=\frac{\left(\bar{T}_{f}-\bar{T}_{c}\right)}{\left(\bar{T}_{h}-\bar{T}_{c}\right)} ; D a=\frac{\bar{k}}{H^{2}} ; y=\frac{\bar{y}}{H} ; d=\frac{\bar{d}}{H} ; \\
& U_{p}=\frac{v \bar{U}_{p}}{g \beta H^{2}\left(\bar{T}_{h}-\bar{T}_{c}\right)}, \theta_{p}=\frac{\left(\bar{T}_{p}-\bar{T}_{c}\right)}{\left(\bar{T}_{h}-\bar{T}_{c}\right)} ; N=\frac{g^{2} \beta^{2} H^{4}\left(\bar{T}_{h}-\bar{T}_{c}\right)}{k v} ; M=\frac{\sigma B_{0}^{2} h^{2}}{v}
\end{aligned}
$$

Withreference to the problem underconsideration, the basic equations in non-dimensional quantities for the equations (1) to (5) can be expressed as:

Fluid region 


$$
\begin{aligned}
& \frac{d^{2} U_{f}}{d y^{2}}+\theta_{f}-M U_{f}=0 \\
& \frac{d^{2} \theta_{f}}{d y^{2}}+N\left(\frac{d U_{f}}{d y}\right)^{2}=0
\end{aligned}
$$

Porous Region

$$
\begin{aligned}
& \frac{d^{2} U_{p}}{d y^{2}}+\theta_{p}-\left(M+\frac{1}{D a}\right) U_{p}=0 \\
& \frac{d^{2} \theta_{p}}{d y^{2}}+N\left(\frac{d U_{p}}{d y}\right)^{2}+\frac{N}{D a} U_{p}^{2}=0
\end{aligned}
$$

The acquired dimensionless forms of the boundary conditions are:

$$
\begin{array}{llr}
U_{f}=u_{0} ; \theta_{f}=A & \text { at } & y=0 \\
U_{p}=-u_{0} ; \theta_{f}=B & \text { at } & y=1 \\
\frac{d U_{f}}{d y}=\frac{d U_{p}}{d y} ; U_{f}=U_{p} & \text { at } & y=d \\
\frac{d \theta_{f}}{d y}=\frac{d \theta_{p}}{d y} ; \theta_{f}=\theta_{p} & \text { at } & y=d
\end{array}
$$

\section{SOLUTION METHODOLOGY}

The above problem considered is non-linear and can be solved by perturbation technique.Most often in practical problems, $\mathrm{N}$ is assumed to be small. Consider the expansion:

$$
\begin{array}{ll}
U_{f}=U_{0 f}+N U_{1 f}+O\left(N^{2}\right) & \theta_{f}=\theta_{0 f}+N \theta_{1 f}+O\left(N^{2}\right) \\
U_{p}=U_{0 p}+N U_{1 p}+O\left(N^{2}\right) & \theta_{p}=\theta_{0 p}+N \theta_{1 p}+O\left(N^{2}\right)
\end{array}
$$

Introducing (12) in equation (7) to equation (10), we get

$\frac{d^{2} U_{0 f}}{d y^{2}}-M U_{0 f}+\theta_{0 f}=0$

$\frac{d^{2} U_{1 f}}{d y^{2}}-M U_{1 f}+\theta_{1 f}=0$

$\frac{d^{2} \theta_{0 f}}{d y^{2}}=0$

$\frac{d^{2} \theta_{1 f}}{d y^{2}}+\left(\frac{d U_{0 f}}{d y}\right)^{2}=0$

$\frac{d^{2} U_{0 p}}{d y^{2}}-\left(\frac{1}{D a}+M\right) U_{0 p}+\theta_{0 p}=0$

$\frac{d^{2} U_{1 p}}{d y^{2}}-\left(\frac{1}{D a}+M\right) U_{1 p}+\theta_{1 p}=0$

$\frac{d^{2} \theta_{0 p}}{d y^{2}}=0$

$\frac{d^{2} \theta_{1 p}}{d y^{2}}+\left(\frac{d U_{0 p}}{d y}\right)^{2}+\frac{1}{D a} U_{0 p}^{2}=0$

The final boundary conditions are: 


$$
\begin{array}{lll}
U_{1 f}=0 ; U_{0 f}=u_{0} ; \theta_{1 f}=0 ; \theta_{0 f}=A & \text { at } & y=0: \\
U_{1 p}=0 ; U_{0 p}=-u_{0} ; \theta_{1 p}=0 ; \theta_{0 p}=B ; & \text { at } & y=1: \\
U_{1 f}=U_{1 p} ; U_{0 f}=U_{0 p} ; \frac{d U_{1 f}}{d y}=\frac{d U_{1 p}}{d y} ; \frac{d U_{0 f}}{d y}=\frac{d U_{0 p}}{d y} & \text { at } & y=d ; \\
\theta_{1 f}=\theta_{1 p} ; \theta_{0 f}=\theta_{0 p} ; \frac{d \theta_{0 f}}{d y}=\frac{d \theta_{0 p}}{d y} ; \frac{d \theta_{1 f}}{d y}=\frac{d \theta_{1 p}}{d y} & \text { at } & y=d
\end{array}
$$

The solution for equations (13) - (20) are obtained by using the boundary conditions (21).

$$
\begin{aligned}
& U_{0 f}=a_{1} e^{\sqrt{M} y}+a_{2} e^{-\sqrt{M} y}+\frac{A+(B-A) y}{M} \\
& U_{0 p}=a_{5} e^{\sqrt{K} y}+a_{6} e^{-\sqrt{K} y}+\left(\frac{A+(B-A) y}{K}\right) \\
& \theta_{0 f}=\theta_{0 f}=(A)+(B-A) y \\
& \theta_{1 f}=\frac{-a_{1}^{2} e^{2 \sqrt{M} y}}{4}+\frac{a_{2}^{2} e^{-2 \sqrt{M} y}}{4}-\frac{(B-A)^{2} y^{2}}{2 M^{2}}+a_{1} a_{2} M y^{2}+\frac{2 a_{2} e^{-\sqrt{M} y}}{M \sqrt{M}}(B-A)-\frac{2 a_{1} e^{\sqrt{M} y}}{M \sqrt{M}}(B-A) \\
& -K_{1} y-K_{2} \\
& \theta_{1 p}=\frac{a_{5}^{2} K_{3} e^{2 \sqrt{K} y}}{4 K}+\frac{a_{6}^{2} K_{3} e^{-2 \sqrt{K} y}}{4 K}+\frac{K_{4} y}{K^{2}}+2 a_{5} a_{6} K_{3} \frac{y^{2}}{2}+\frac{A+(B-A) y}{D a K} \frac{2\left(a_{5}+a_{6}\right) e^{-\sqrt{K} y}}{K} \\
& +\frac{(B-A)}{K} \frac{2\left(a_{5}+a_{6}\right) e^{\sqrt{K} y}}{\sqrt{K}}+K_{5} y+K_{6} \\
& U_{1 f}=a_{3} e^{\sqrt{M} y}+a_{4} e^{-\sqrt{M} y}+\frac{1}{M}\left[\begin{array}{l}
\frac{-a_{1}^{2} e^{2 \sqrt{M} y}}{4}+\frac{a_{2}^{2} e^{-2 \sqrt{M} y}}{4}-\frac{(B-A)^{2} y^{2}}{2 M^{2}}+a_{1} a_{2} M y^{2}+ \\
\frac{2 a_{2} e^{-\sqrt{M} y}}{M \sqrt{M}}(B-A)-\frac{2 a_{1} e^{\sqrt{M} y}}{M \sqrt{M}}(B-A)-K_{1} y-K_{2}
\end{array}\right] \\
& U_{1 p}=a_{7} e^{\sqrt{K} y}+a_{8} e^{-\sqrt{K} y}+\frac{1}{K}\left[\begin{array}{l}
\frac{a_{5}^{2} K_{3} e^{2 \sqrt{K} y}}{4 K}+\frac{a_{6}^{2} K_{3} e^{-2 \sqrt{K} y}}{4 K}+\frac{K_{4} y}{K^{2}}+2 a_{5} a_{6} K_{3} \frac{y^{2}}{2}+ \\
\frac{A+(B-A) y}{D a K} \frac{2\left(a_{5}+a_{6}\right) e^{-\sqrt{K} y}}{K} \\
+\frac{(B-A)}{K} \frac{2\left(a_{5}+a_{6}\right) e^{\sqrt{K} y}}{\sqrt{K}}+K_{5} y+K_{6}
\end{array}\right]
\end{aligned}
$$

Where

$$
\begin{aligned}
& a_{1}=u_{0}-a_{2}-\frac{A}{M} \\
& a_{2}=\sinh ^{-1}\left[\left(u_{0}-\frac{A}{M}\right) e^{\sqrt{M} d}+A+(B-A) d\left[\frac{1}{M}+\frac{1}{K}\right]-a_{5} e^{\sqrt{K} d}-a_{6} e^{-\sqrt{K} d}\right] \\
& a_{3}=-\left[a_{4}+\frac{1}{M}\left[\frac{\left(a_{2}^{2}-a_{1}^{2}\right)}{4}-\frac{2 a_{2}}{M \sqrt{M}}(B-A)-\frac{2 a_{1}}{M \sqrt{M}}(B-A)-K_{2}\right]\right] \\
& a_{4}=e^{\sqrt{M} d}\left[a_{2} e^{\sqrt{K} d}+a_{2} e^{-\sqrt{K} d}+\frac{1}{K}\left(K_{6}-K_{8}\right)-\frac{K_{7}}{M}\right] \\
& a_{5}=\frac{e^{-\sqrt{K} d}}{\sqrt{K}}\left[K_{15}\right] \\
& a_{6}=e^{\sqrt{K}}\left[K_{16}\right]
\end{aligned}
$$




$$
\begin{aligned}
& a_{7}=\left[\frac{K_{9}}{K}-a_{8} e^{-\sqrt{K}}\right] e^{-\sqrt{K}} \\
& a_{8}=\frac{e^{\sqrt{K} d}}{\sqrt{K}}\left[K_{10}-K_{11}\right] \\
& K_{1}=K_{14}-K_{12} \\
& K_{2}=\frac{-a_{1}^{2}+a_{2}^{2}}{4}+\frac{2(B-A)}{M \sqrt{M}}\left(a_{2}-a_{1}\right) \\
& K_{5}=K_{9}-K_{6} \\
& K_{6}=K_{7}+K_{8} \\
& K_{7}=\frac{-a_{1}^{2} e^{2 \sqrt{M} d}}{4}+\frac{a_{1}^{2} e^{-2 \sqrt{M} d}}{4}-\frac{(B-A)^{2}}{2 M^{2}} d^{2}+a_{1} a_{2} M d^{2}+\frac{2 a_{2} e^{-\sqrt{M} d}}{M \sqrt{M}}(B-A)-\frac{2 a_{1} e^{\sqrt{M} d}}{M \sqrt{M}}(B-A)-K_{1} d-K_{2} \\
& K_{8}=-\frac{a_{5}^{2} K_{3} e^{2 \sqrt{K} d}}{4 K}-\frac{a_{6}^{2} K_{3} e^{-2 \sqrt{K} d}}{4 K}-\frac{K_{4}}{K^{2}} d-a_{5} a_{6} K_{3} d^{2}-\frac{A+(B-A) d}{D a} \frac{\left(2\left(a_{5}+a_{6}\right)\right) e^{-\sqrt{K} d}}{K^{2}} \\
& -\frac{\left(2\left(a_{5}+a_{6}\right) e^{\sqrt{K} d}\right)}{(\sqrt{K})} \frac{(B-A)}{K}-K_{5} d \\
& K_{9}=\frac{1}{K}\left[\begin{array}{rr}
-\frac{a_{5}^{2} K_{3} e^{2 \sqrt{K}}}{4 K}-\frac{a_{6}^{2} K_{3} e^{-2 \sqrt{K}}}{4 K}-\frac{K_{4}}{K^{2}}-a_{5} a_{6} K_{3}-\left(\frac{(A+(B-A) d)}{D a k} \frac{\left.\left(2\left(a_{5}+a_{6}\right)\right) e^{-\sqrt{K}}\right)}{K}\right) \\
-\frac{\left(2\left(a_{5}+a_{6}\right) e^{\sqrt{K}}\right)}{(\sqrt{K})} \frac{(B-A)}{K}-K_{5}+\left(K_{6}\right)
\end{array}\right] \\
& \backslash K_{10}=\frac{1}{K}\left[\begin{array}{c}
-\frac{2 \sqrt{K} a_{5}^{2} K_{3} e^{2 \sqrt{K} d}}{4 K}-\frac{2 \sqrt{K} a_{6}^{2} K_{3} e^{-2 \sqrt{K} d}}{4 K}-\frac{K_{4}}{K^{2}}-2 a_{5} a_{6} K_{3} d-\frac{A+(B-A) d}{D a k} \frac{\left(2\left(a_{5}+a_{6}\right)\right) e^{-\sqrt{K} d}}{-\sqrt{K} K} \\
-\frac{(B-A)}{K} \frac{2\left(a_{5}+a_{6}\right) e^{\sqrt{K} d}}{\sqrt{K}}-K_{5}
\end{array}\right] \\
& K_{11}=\frac{1}{M}\left[\begin{array}{rr}
-\frac{2 \sqrt{M} a_{1}^{2} e^{2 \sqrt{M} d}}{4}-\frac{2 \sqrt{M} a_{2}^{2} e^{-2 \sqrt{M} d}}{4}-\frac{(B-A)^{2}}{M^{2}} d+2 a_{1} a_{2} M d-\frac{(B-A)}{M^{2}} \frac{2 a_{2} e^{-\sqrt{M} d}}{K} \\
-\frac{(B-A)}{M^{2}} \frac{2 a_{1} e^{\sqrt{M} d}}{M^{2}}-K_{1}
\end{array}\right] \\
& K_{12}=\sqrt{M} a_{3} e^{\sqrt{M} d}-\sqrt{M} a_{4} e^{-\sqrt{M} d}-\sqrt{K} a_{7} e^{\sqrt{K} d} \\
& K_{13}=K_{11}+K_{12} \\
& K_{14}=\left[M K_{11}\right] \\
& K_{15}=\left[\sqrt{K} a_{6} e^{-\sqrt{K} d}+\sqrt{M}\left(a_{1} e^{\sqrt{M} d}-a_{2} e^{-\sqrt{M} d}\right)+(A+(B-A))\left[\frac{1}{M}-\frac{1}{K}\right]\right] \\
& K_{16}=\left[u_{0}-a_{5} e^{\sqrt{K}}-\frac{A+(B-A)}{K}\right]
\end{aligned}
$$

\section{RESULTS AND ANALYSIS}

To discuss the physical significance of important parameters of flow, 'MATLAB' has been used.

Assuming that the infinite vertical channelismoving in a opposite direction,the partly loaded porous medium has been analyzed in order to mull over the physical configuration of the laminar Mhd fully developed flow.The momentum and energy equation solutions are obtained by adopting perturbation technique, and the findings are presented graphically for principleparameters of the flow region.The zero order and first order velocity and temperature distribution are presented for different cases. Due to the linearity, the zero-order temperature profile is not considered for discussion.

Theclear fluid aty $=0$, interface fluid at region near $\mathrm{y}=\mathrm{d}$ andporous regionat $\mathrm{y}=1$ have been illustrated.

Case (i) The plate $\mathrm{y}=0$ is heated, when $\mathrm{A}=1$ and the 
plate $\mathrm{y}=1$ is cooled, when $\mathrm{B}=0$.

Case(ii) The plate $\mathrm{y}=0$ is cooled, when $\mathrm{A}=1$ and $\mathrm{y}=1$ is heated, when $\mathrm{B}=1$.

Fig 1.1 to Fig 1.3 depicts the velocity profile $\left(\mathrm{U}_{\text {of }}\right.$ or $\mathrm{U}_{\mathrm{op}}$ ), for case (i) with different parameters Darcy number (Da),Magnetic field parameter (M) and width (d).Itis observed that the velocity profile is decreasedwith the upgrading values ofDa and $\mathrm{M}$. It is noted that the graph degrades with increasing $\mathrm{d}$.

Fig 1.4 to fig. 1.6 illustrates the velocity distribution profile $\left(\mathrm{U}_{\text {of }}\right.$ or $\left.\mathrm{U}_{\mathrm{op}}\right)$ for case(ii).Theprofile seems to decrease for incrementing values of $\mathrm{Da}$ and $\mathrm{M}$ but the profile is elevated for ascending values of $\mathrm{d}$.

From fig 2.1 to fig.2.6 it is observed that the velocity $\left(\mathrm{U}_{1 \mathrm{f}}\right.$ or $\left.\mathrm{U}_{1 \mathrm{p}}\right)$ flow proliferates with increasing $\mathrm{M}$ and $\mathrm{d}$ for either A or B exists. But the Darcy number Da decelerates the velocity flow for case (i) and it accelerates the flow for case (ii)

From fig 3.1 to fig. 3.6 it is observed that the effect of the parameters $\mathrm{M}$ and $\mathrm{Da}$. It is noted that the temperature distribution of the flow enhances for either cases. But for increasing $d$ values, it is noted that the temperature profiledeceleratesfor case (i) and accelerates for case (ii).

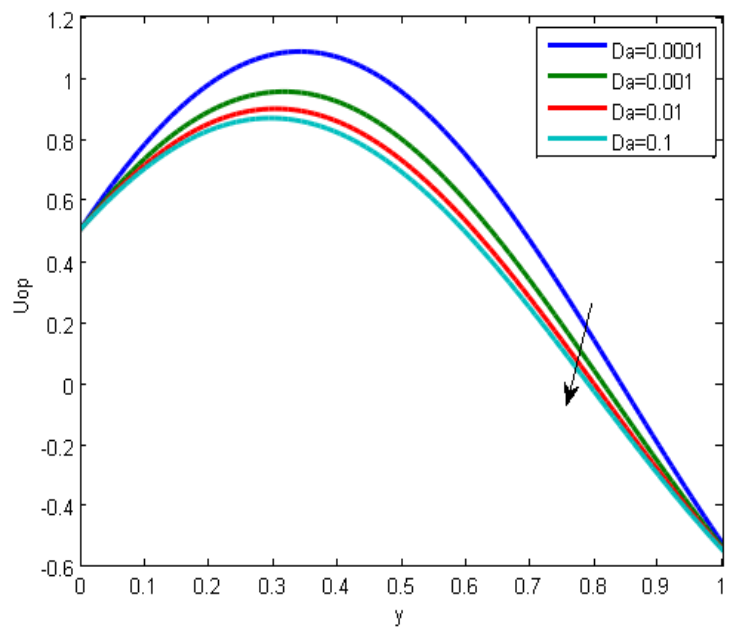

Fig 1.1: Velocity profile for Da, $[\mathrm{A}=1 ; \mathrm{B}=0 ; \mathrm{u} 0=0.5 ; \mathrm{d}=0.3 ; \mathrm{M}=2]$

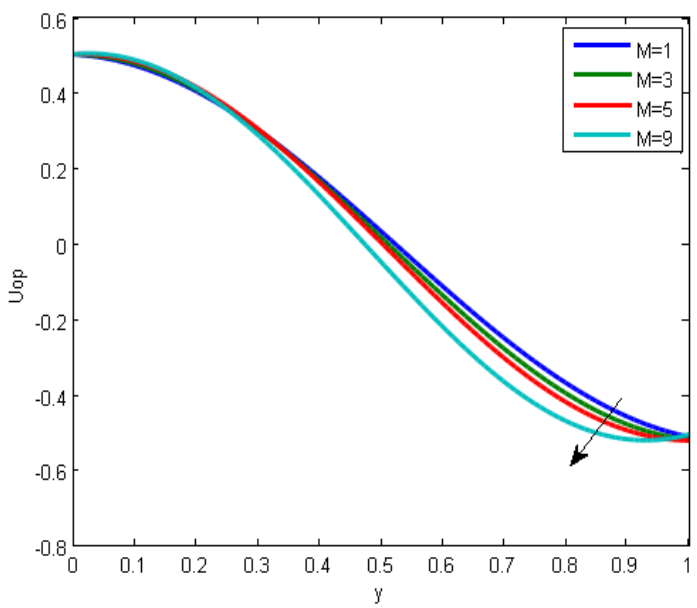

Fig 1.2: Velocity profile for $\mathrm{M},[\mathrm{A}=1 ; \mathrm{B}=0 ; \mathrm{u} 0=0 ; \mathrm{d}=0.3 ; \mathrm{Da}=0.1]$

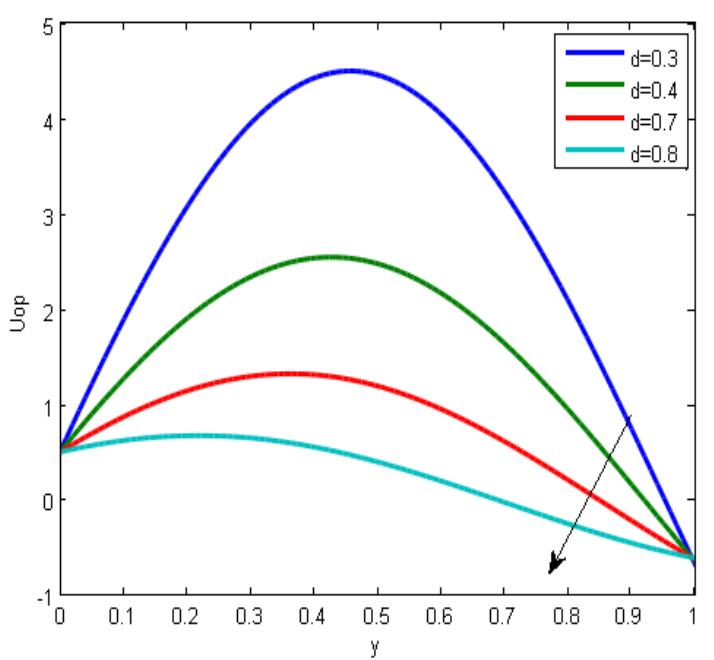

Fig 1.3: Velocity stetch for $\mathrm{d}:[\mathrm{Da}=0.1$; $\mathrm{A}=1 ; \mathrm{B}=0 ; \mathrm{u} 0=0.5 ; \mathrm{M}=2]$

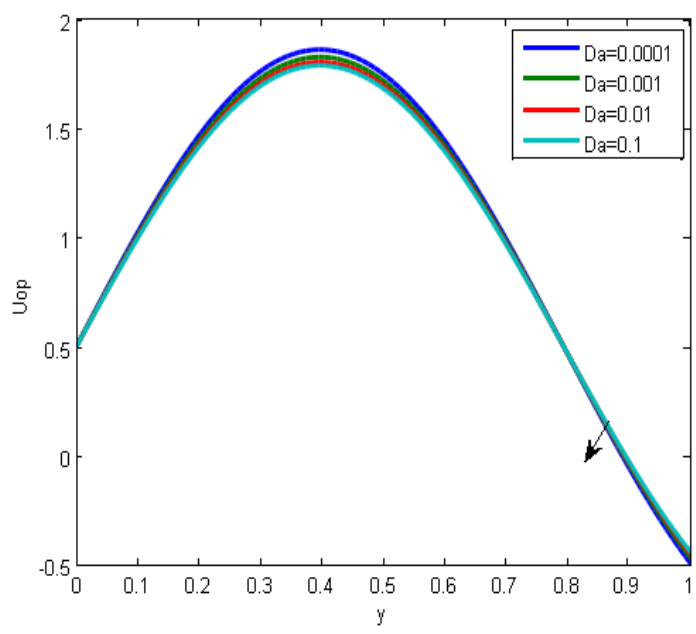

Fig 1.4: Velocity profile for $\mathrm{Da}:[\mathrm{A}=0 ; \mathrm{B}=1 ; \mathrm{u} 0=0.5 ; \mathrm{d}=0.3 ; \mathrm{M}=2]$

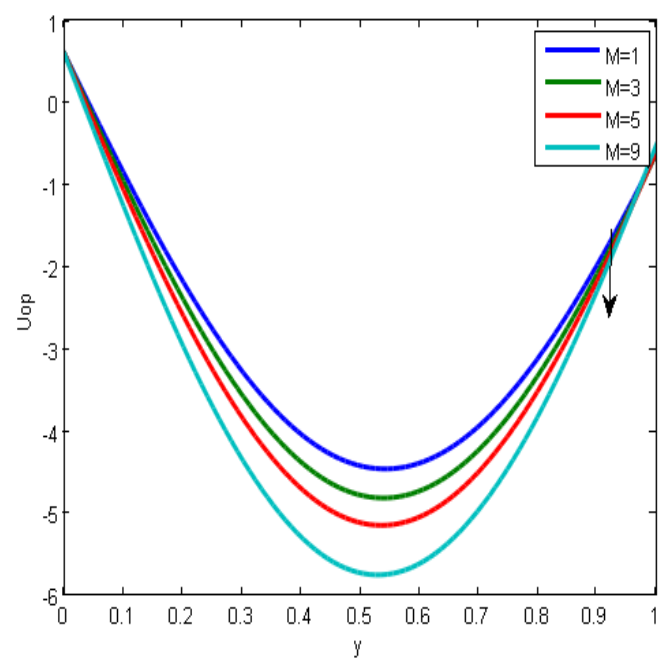

Fig 1.5: Graph of velocity Uop for $\mathrm{M}$ : $[\mathrm{Da}=0.1$, $\mathrm{A}=0 ; \mathrm{B}=1 ; \mathrm{u} 0=0.5 ; \mathrm{d}=0.3$ ] 


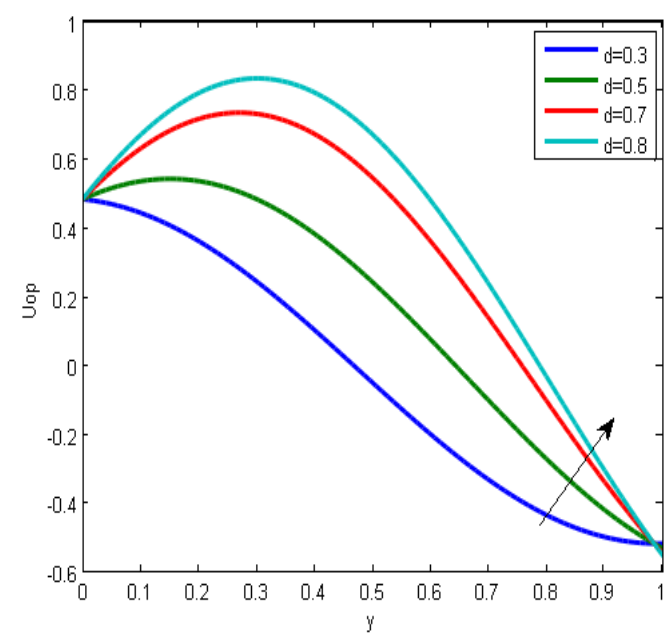

Fig 1.6: Graph of velocity Uop for $\mathrm{d}$ : $[\mathrm{Da}=0.1$, $\mathrm{A}=0 ; \mathrm{B}=1 ; \mathrm{u} 0=0.5 ; \mathrm{M}=2]$

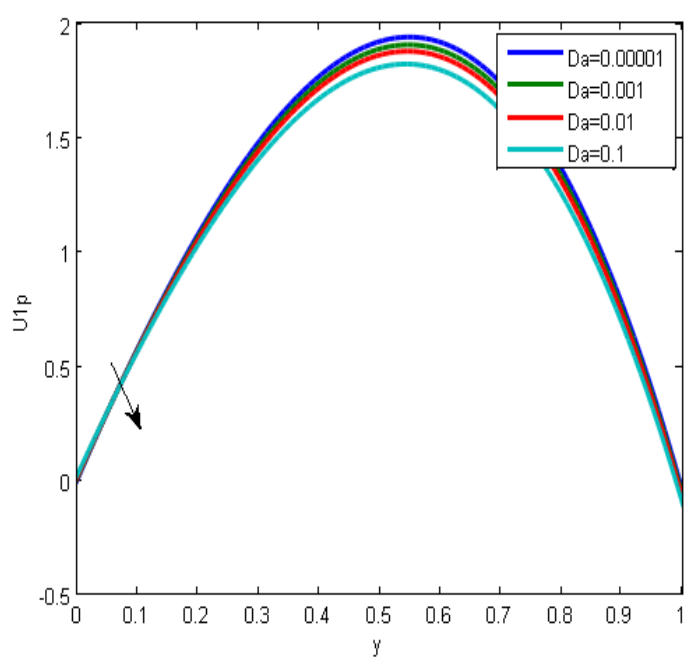

Fig 2.1: Graph of velocity U1p for Da; $[\mathrm{M}=2$, $\mathrm{A}=1 ; \mathrm{B}=0 ; \mathrm{u} 0=0.5 ; \mathrm{d}=0.3$ ]

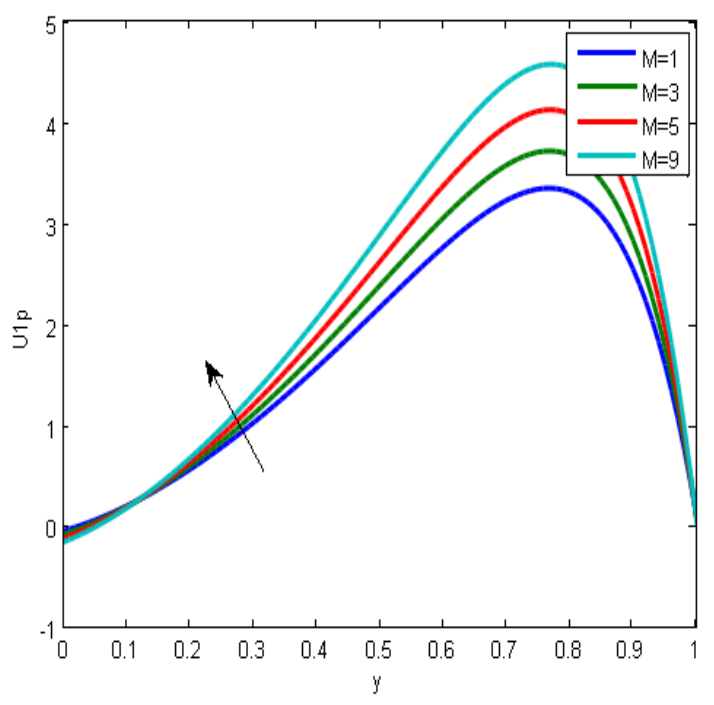

Fig 2.2: Profile of velocity U1p for $\mathrm{M}$; $[\mathrm{Da}=0.1$, $\mathrm{A}=1 ; \mathrm{B}=0 ; \mathrm{u} 0=0.5 ; \mathrm{d}=0.3]$

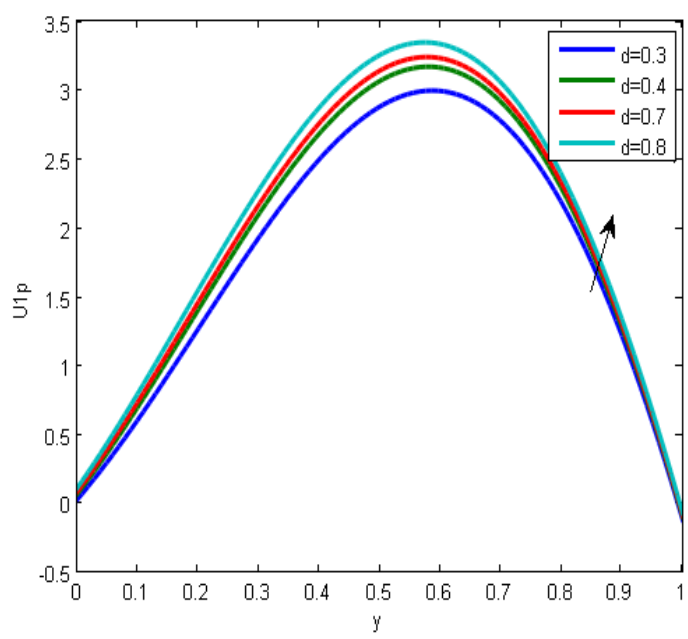

Fig 2.3: Graph of velocity U1p for $\mathrm{d}$; $[\mathrm{M}=2$, $\mathrm{A}=1 ; \mathrm{B}=0 ; \mathrm{u} 0=0.5 ; \mathrm{Da}=0.1]$

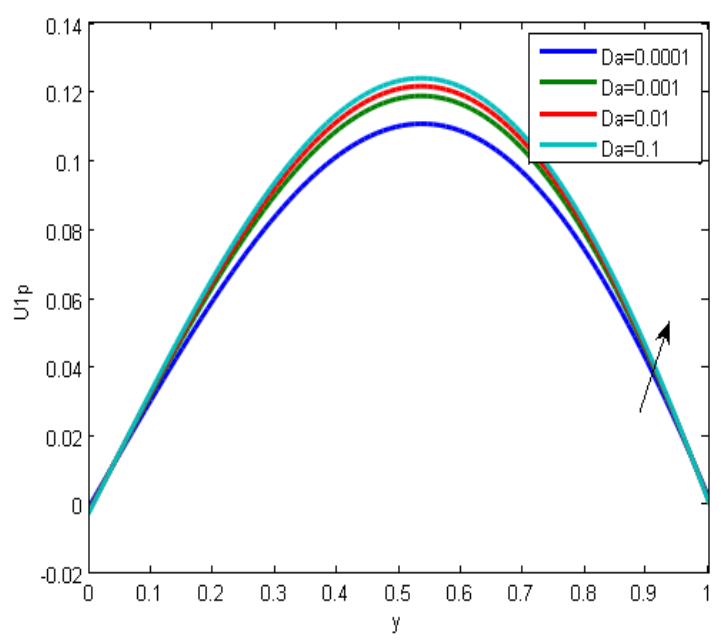

Fig 2.4: Graph of velocity U1p for Da; $[\mathrm{M}=2, \mathrm{~A}=0 ; \mathrm{B}=1 ; \mathrm{u} 0=0.5 ; \mathrm{d}=0.3]$

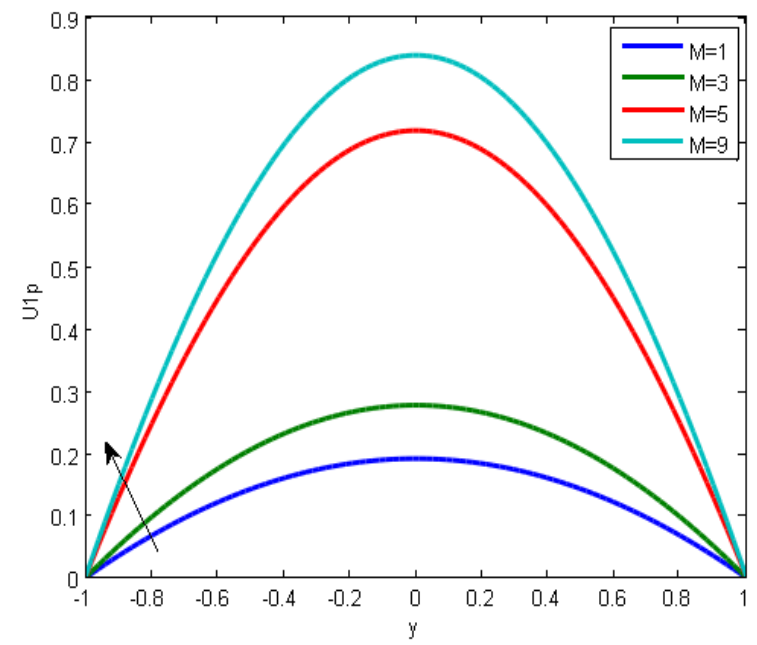

Fig 2.5: Profile of velocity U1p for $\mathrm{M}$; $[\mathrm{Da}=0.1$, $\mathrm{A}=0 ; \mathrm{B}=1 ; \mathrm{u} 0=0.5 ; \mathrm{d}=0.3]$

Published By:

Blue Eyes Intelligence Engineering \& Sciences Publication 
The Influence of Inclined Magnetic Field and Heat Transfer on the MHD Convective Flow in a Vertical Channel Filled Partly with Porous Medium

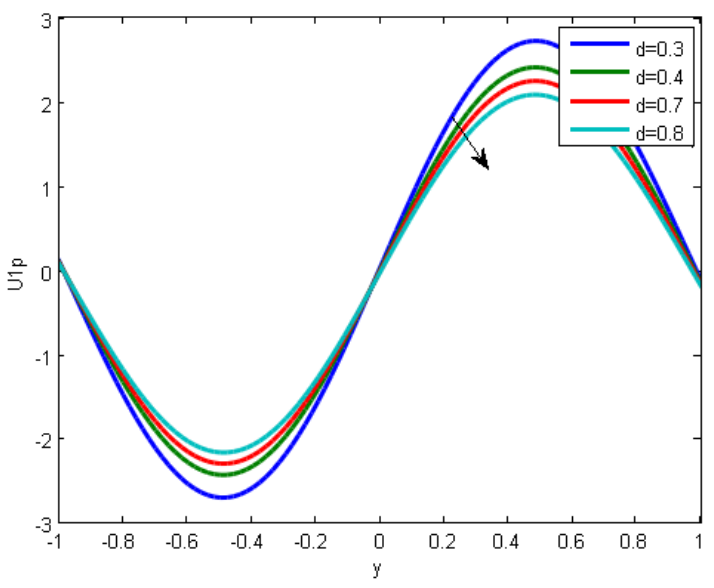

Fig 2.6: stetch of velocity U1p for d; $[\mathrm{M}=2, \mathrm{~A}=0 ; \mathrm{B}=1 ; \mathrm{u} 0=0.5 ; \mathrm{Da}=0.1]$

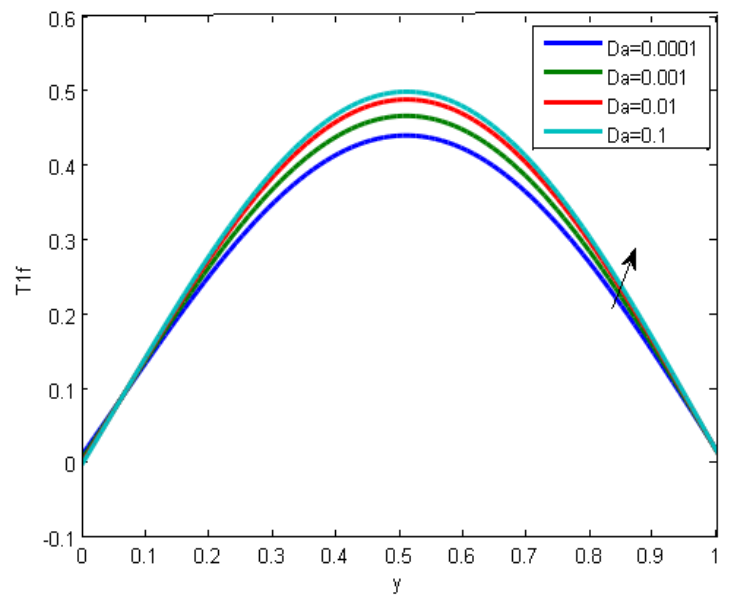

Fig 3.1: Graph of temperature T1f for $\mathrm{Da} ;[\mathrm{M}=2$, $\mathrm{A}=1 ; \mathrm{B}=0 ; \mathrm{u} 0=0.5 ; \mathrm{d}=0.3$ ]

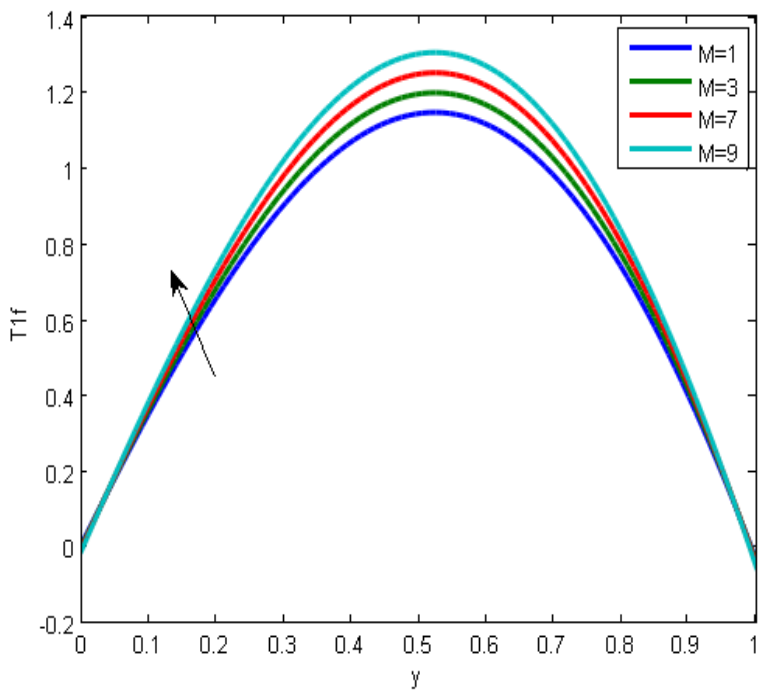

Fig 3.2: Graph of temperature T1f for $M$; $[\mathrm{M}=2$, $\mathrm{A}=1 ; \mathrm{B}=0 ; \mathrm{u} 0=0.5 ; \mathrm{Da}=0.1]$

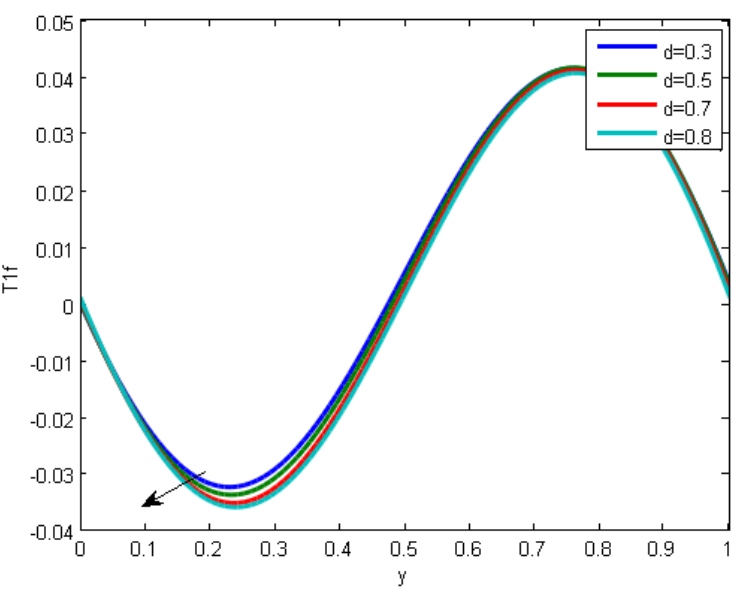

Fig 3.3: Stetch of temperature T1f for $d ;[M=2$, $\mathrm{A}=1 ; \mathrm{B}=0 ; \mathrm{u} 0=0.5 ; \mathrm{Da}=0.1]$

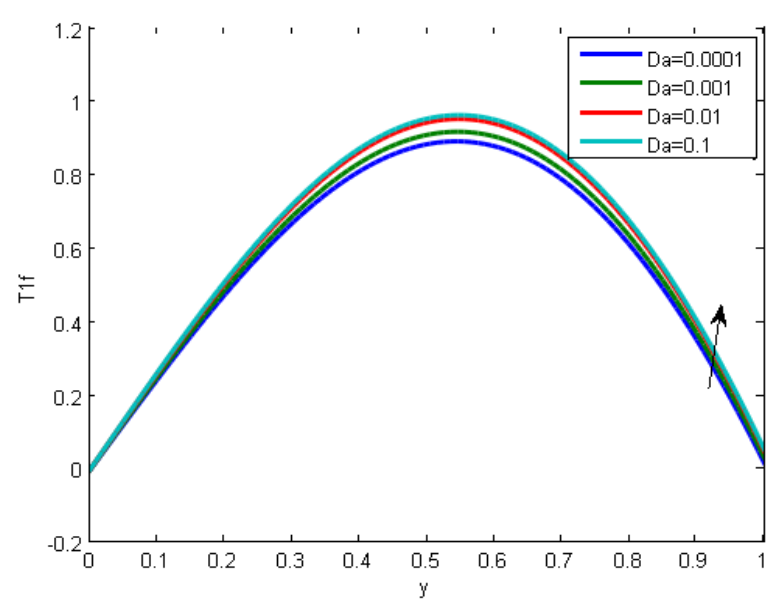

Fig 3.4:Temperature profile T1f for Da; $[\mathrm{M}=2$, $\mathrm{A}=0 ; \mathrm{B}=1 ; \mathrm{u} 0=0.5 ; \mathrm{d}=0.3]$

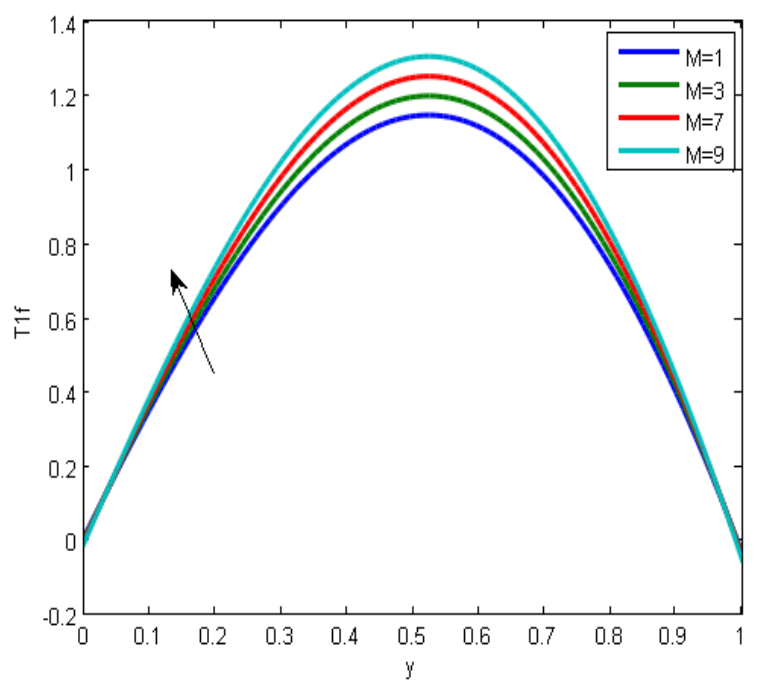

Fig 3.5: Stetch of temperature T1f for $\mathrm{M}$; $[\mathrm{d}=0.3$, $\mathrm{A}=0 ; \mathrm{B}=1 ; \mathrm{u} 0=0.5 ; \mathrm{Da}=0.1]$

Published By: 


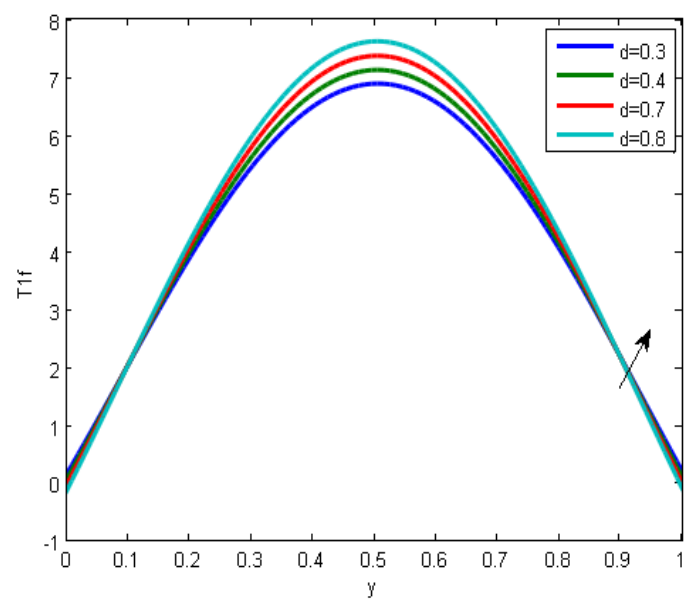

Fig 3.6: Graph of temperature T1f for $\mathrm{d}$ : $[\mathrm{M}=2$, $\mathrm{A}=0 ; \mathrm{B}=1 ; \mathrm{u} 0=0.5 ; \mathrm{Da}=0.1]$

\section{DISCUSSION AND CONCLUSION}

The analysis of a laminar fully developed Mhd flow bounded by vertically parallel plates which are moving in opposite direction and maintained at different temperature in presence of inclined magnetic field has been studied. The expression for velocity and temperature are obtained by implementing perturbation technique.It is imperative to conclude that for case (i) the velocity profile $\left(\mathrm{U}_{0 \mathrm{f}}\right.$ or $\left.\mathrm{U}_{0 \mathrm{p}}\right)$ degrades for incrementing values of parameters $\mathrm{d}, \mathrm{M}$ and Da.Whereas in case (ii) the velocity profile shows reduction with inclusion of $\mathrm{M}$ and $\mathrm{Da}$ but it accelerates with the increasing $\mathrm{d}$ value.On the other hand, the velocity profile $\left(U_{1 f}\right.$ or $\left.U_{1 p}\right)$ enhances the flow for case (i) with variation of $\mathrm{M}, \mathrm{d}$ and it seems to decrease for increasing $\mathrm{Da}$ value. In case (ii) the velocity profile progresses for ascending values of $\mathrm{M}$ and $\mathrm{Da}$ but it decreases for increasing $\mathrm{d}$ values. As per the temperature profile is concerned, the profile $\left(\theta_{1 \mathrm{ff}}\right.$ or $\left.\theta_{1 \mathrm{p}}\right)$ for case(i) it seems to decrease by incrementing $\mathrm{d}$ values andincreasesfor increasing magnetic field $\mathrm{M}$ and darcy number $\mathrm{Da}$. It is notedfrom case (ii) that the temperature profile communicates the same increasing behavior with the predominant parameters $\mathrm{Da}, \mathrm{M}$ and $\mathrm{d}$.

\section{REFERENCES}

1. M.M.S. El-Din, "Laminar fully developed mixed convection with viscous dissipation in a uniformly heated vertical double-passage channel", Int. J. of Therm. Sci., 11(1), 2007, 27-41

2. T. Paul and A. K. Singh, "Transient natural convection between two vertical walls heated/cooled asymmetrically", Int. J. Applied Mechanics and Engineering, 11(1), 2006, 143-154.

3. N.B. Reddy ,V.R. Prasad, "Radiation effects on an unsteady MHD convective heat and mass transfer flow past a semi-infinite vertical permeable moving plate embedded in a porous medium", J. Energy, Heat and Mass Transfer, 30(2008), 57-78. V. Kumar and D.S. Chauhan, "Effects of slip conditions on forced convection and entropy generation in a circular channel occupied by a highly porous medium: Darcy extended Brinkman-Forchheimer model", Turkish J. Eng. Env. Sci., 33,2009, 91-104.

4. N. Srivastava and A. K. Singh, "Mixed Convection in a Composite System Bounded by Vertical Walls", Journal of Applied Fluid Mechanics, 3(2), 2010, 65-75.

5. Abhay Kumar Jha, Umesh Gupta, Rama Charan Chaudhary, "Free Convection Flow between Vertical Plates Moving in Opposite Direction and Partially Filled with Porous Medium", Applied Mathematics, 2, 2011, 935-941.

6. Dileep Singh Chauhan and Rashmi Agrawal, "Magnetohydrodynamic Convection Effects with Viscous and Ohmic Dissipation in a Vertical
Channel Partially Filled by a Porous Medium", Journal of Applied Science and Engineering, 15, 2012, 1-10.

7. R. Choudhary and S. Purkayastha, "Visco-elastic effects on convection flow in a vertical rotating channel partially filled with a porous medium", Int. J. of Computer Applications, 104, 2014, 9-17. https://doi.org/10.5120/18189-9098 Prasad, K. V., K. Vajravelu, P. S. Datti and B. T. Raju, "MHD Flow and Heat Transfer in a Power-law Liquid Film at a Porous Surface in the Presence of Thermal Radiation", Journal of Applied Fluid Mechanics 6(3), 2013,385-395

8. K.V, Prasad, K.Vajravelu , P. S. Datti and B. T. Raju , "MHD Flow and Heat Transfer in a Power-law Liquid Film at a Porous Surface in the Presence of Thermal Radiation", Journal of Applied Fluid Mechanics 6(3), 2013,385-395

9. Adeyemi IsaiahFagbade, Bidemi OlumideFalodun, Chika Uchechukwu Boneze, "Influence of Magnetic Field, Viscous Dissipation and Thermophoresis on Darcy-Forcheimer Mixed Convection Flow in Fluid Saturated Porous Media", American Journal of Computational Mathematics, 5,2015,18-40

10. Adeniyan. A and Abioye, "Mixed Convection Radiating Flow and Heat Transfer in a Vertical Channel Partially Filled With a DarcyForchheimer Porous Substrate", Gen. Math. Notes, 32(2), 2016, 80104. http://www.geman.in.

11. Dhananjaya .S, P.V. Arunachalam, S. Sreenadh, A. Parandhama , "Free Convection Flow of a Jeffrey Fluid between Vertical Plates Partially Filled with Porous Medium", International Journal of Scientific Research Engineering \& Technology (IJSRET), 4(5), 2015,503-512.

12. Pooja Sharma, Ruchi Saboo, Heat and Mass Transfer in free Convective Flow of Walter's Liquid Model-B through Rotating Vertical Channel, Applied Mathematical Sciences, 11(33), 2017, 1651

13. Gupta V. G, Abhay Kumar Jha, Ajay Jain. "Convective Effects on MHD Flow and Heat Transfer between Vertical Plates Moving in Opposite Direction and Partially Filled with a Porous Medium", Journal of Applied Mathematics and Physics, 4, 2016, 341-358.

\section{AUTHORS PROFILE}

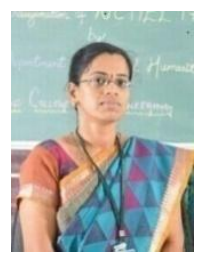

Ms.M.Shanthi. She is perusing Ph.D in the field of fluid dynamics in Karpagam Academy of higher Education and working as an assistant professor, S\&H department in Karpagam College of Engineering. she has published research articles and presented papers in national conference. She has keen interest and a learner in research aspects related to fluid dynamics.

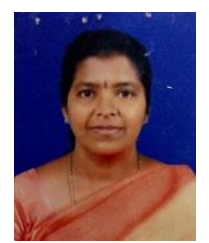

Dr.P.T.Hemamalini.She has completed her Ph.D research work "Ferrofluids-Studies in sability of ferrofluids " in 2008. She is working as a Professor and HOD ( $\mathrm{S} \& \mathrm{H})$ in Karpagam Academy of Higher Education. She has published 7 mathematics books and more than 23 research articles.she has received copy rights for her Braille books(14 volumes) " Self instructional mathematics material for the $\mathrm{X}$ standard students with Visual Impairment". She is honoured with awards like "Bharat Ratna Dr.Abdul Kalam Gold medal award"-2008,:Best teaching excellency award"-2019,"Doctor of letters(D.litt)" \& "Bharitiya udyog Ratan gold medal award" from "Global Economic Progress \& Research Association", etc.She has guided research scholars and her research in the study of ferrofluids are highly commendable. 